\title{
"Speeding up the road to recovery": The Complex Recovery Assessment and Consultation (CRAC) service
}

\author{
Stephanie Davis Le Brun \\ Cheshire and Wirral Partnership NHS Foundation Trust, United Kingdom
}

\begin{abstract}
The number of bed closures in mental health is on the rise, creating additional pressure on services, including acute mental health services. An efficient way of working is required in order to streamline the acute care pathway and decrease unnecessary delays to length of stay, ensuring all individuals can be offered an inpatient bed when in crisis. The Complex Recovery Assessment and Consultation (CRAC) service was created in order to support acute mental health inpatient clinicians in streamlining hospital stays for service users who present with complex presentations that require lengthier admissions (over 40 days) by offering assessment, advice, and intervention from a rehabilitation perspective. The team was also created to understand why individuals may require a lengthy hospital stay.

Preliminary data showed that requiring a placement on discharge proved to be the most significant factor in increased length of stay and so the team took on a new role of discharge coordinator after around a year of operating. This involved assisting in decreasing any delays out of hospital through improved communication and dedicated time to complete tasks, such as completing paperwork for placement referrals and funding panels. Since taking on this role it was found that the time taken for individuals to be discharged to a rehabilitation or specialist placement decreased; a rehabilitation placement by 13.12 days and a specialist placement by 9.22 days. Discharge to a family address also decreased by 2.9 days and a home address by 2.47 days. Those patients with complex presentations benefit from having one dedicated team to coordinate the discharge process. Their lengthier acute inpatient stay is improved through streamlining care pathways, ultimately decreasing delays in discharge.
\end{abstract}

\section{Problem}

Care and treatment in the community works well for the vast majority of people with mental illness. However, some individuals may need access to acute inpatient care at some stage in their illness. Inpatient stays are costly and admission onto an acute ward doesn't always necessarily deliver long term improvement towards recovery, with some people requiring repeat admissions in the future. For many the acute care model works well and can have successful outcomes, however it seems that for a number of individuals, for example those with more complex issues, the acute care pathway may not wholly cater to their needs and they may require lengthier hospital admissions. In order for more successful outcomes within acute care, pathways need to be flexible in order to provide individual, holistic care. These pathways also need to be streamlined in order to avoid excess delays on acute wards, which can be frustrating for the individual waiting to be discharged and costly for services, as well as blocking beds for others that may require an acute inpatient stay.

\section{Background}

In October 2013 Community Care published an article around the growing concerns at the number of hospital beds that have been cut in England's mental health services, in particular the National Health Service (NHS). Government policies have focused on intervening early to avoid crisis (Department of Health, 2011) and for more individuals to receive treatment in their homes rather than in hospital (Tyrer, 2011). Over the past two years psychiatric inpatient bed numbers have reduced by approximately 1,700 , around a 9\% reduction (McNicoll, 2013).

The effects of these closures can be significant to those in crisis, often meaning that they cannot be offered admission into hospital when required (Care Quality Commission, 2014), seeing individuals being admitted with far more complex issues, and more likely to be under the framework of the Mental Health Act (Health and Social Care Information Centre, 2013) due to not receiving treatment at the most appropriate time, and to ensure hospital treatment. One knock-on-effect of this is that for these individuals recovery time may become longer due to the longevity of relapse without acute treatment, resulting in reduced throughput on hospital wards (Keown et al, 2007); only adding to the issues outlined.

Until this wider issue can be resolved, services must adapt to be able to support as many individuals as possible within a given time, treating individuals in an efficient and streamlined manner in order for there to be sufficient movement within psychiatric hospital wards. However, it is crucial that the quality of service whilst achieving this must not be compromised.

In 2007, the Care Services Improvement Partnership alongside the National Institute for Mental Health in England published a document outlining good practice in improving discharge from inpatient mental health care. It highlights the need for processes to be streamlined through changing practice and for services to adopt whole system working to improve understanding. When discussing 
discharge planning it recommends that social circumstances should be addressed, information systems should be in place to reduce discharge delays, arrangements with local accommodation providers be made, to introduce new roles to address any issues in this area and to focus on a recovery model of working.

One example of good practice outlined in the document is a new 'discharge facilitator' role in Sheffield Care Trust which aimed to coordinate the discharges of those who found themselves having accommodation or financial issues, to address their needs before they were ready to be discharged. This new role resulted in a $40 \%$ decrease of service users experiencing a delayed discharge.

\section{Baseline measurement}

In 2011 a bed utilisation review was conducted using a point prevalent design. This review looked at all individuals ( 375 separate people from acute admission to discharge) utilising an acute inpatient stay within the Trust on two comparable dates; one date in 2010 and one date in 2011. The review showed that around $30 \%$ of all acute inpatients required more than a brief spell in hospital and/or had needs that were outside of standard acute care. It seemed that a length of stay of around 40 days was the tipping point for this, with those requiring an admission of 40 days or more often having needs that were over and above the support that the acute care model could offer. These individuals often required input from external agencies (eg housing agencies, longer-term/specialist placements) or support from a wider professional network in order to progress in their recovery. Without this it became clear that there may be further need for longer-term, more complex and generally more expensive care in the future.

\section{Design}

The Complex Recovery Assessment and Consultation (CRAC) service was designed following on from the bed utilisation review. The team was built from the existing local rehabilitation unit, which was showing successful outcomes with complex cases; stepping down most individuals into a community placement and showing very low readmission rates. The team is multi-disciplinary, led by an occupational therapist and also comprising of mental health nurses, an assistant psychologist and psychiatrist.

The team focuses on recovery and aims to assess and consult on individuals in acute care from a rehabilitation perspective who have had a length of stay for $40+$ days. The assessment takes a semistructured interview style and explores hopes and goals for the future, strengths of the individual, how they view their circumstances, whether they fully understand their current treatment, their support networks, lifestyle and accommodation. Recommendations are then made to the acute care team from a rehabilitation perspective, whether it be recovery focussed interventions, advising on placements, setting up community support networks etc.

In doing this the team hoped to offer a flexible approach towards the acute care model when appropriate and offer specific recovery focussed intervention where it may not otherwise be accessible (due to nursing time constraints, funding issues etc). The team also hoped to streamline certain processes through effective communication so as to not cause unnecessary delay for discharge, achieving this by having close links with community support providers, independent placement providers and commissioners.

\section{Strategy}

For the first year the team was achieving its aim of assessing those with a length of stay of $40+$ days and offering intervention and consultation when appropriate to do so, assessing 84 individuals during this time (around $56 \%$ of admissions exceeding 40 days). The acute care team could see the benefit of the work and a picture of the demographics of this population was starting to be built; $58 \%$ were male, the average age was 44 years, $88 \%$ stated they were white British, $82 \%$ were open to a community mental health team (CMHT) before admission and $73 \%$ were detained under a Section of the Mental Health Act during some point of their admission. Thirtyeight percent of individuals had been given a diagnosis of paranoid schizophrenia, $24 \%$ a bipolar disorder, $15 \%$ a schizoaffective disorder, $8 \%$ with an episode of psychosis, $7 \%$ with schizophrenia (eg unspecified, other etc), 6\% with a depressive disorder and $2 \%$ with a delusional disorder. Data were (and continues to be) collected looking into the reasons why these individuals may need more than a brief acute stay.

Preliminary analyses on the reasons why these individuals were requiring a hospital admission exceeding 40 days (including; high risk issues, medication review, required placement and/or package of care, non-compliance with medication, lack of engagement, required a spell on the psychiatric intensive care unit (PICU), mental health assessment, physical health problems, delay in diagnosis, required ECT treatment etc) inputted alongside demographic data showed the significant correlating factors as being; requiring placement and/or package of care $(p<0.001)$, previous number of bed days (within the last three years) $(p<0.01)$, Mental Health Act status $(p<0.05)$ and discharge destination $(p<0.05)$.

Forty-nine percent of those with a length of stay exceeding 40 days were not able to go back home/to their original placement, with $14 \%$ being deemed as requiring inpatient rehabilitation, $16 \%$ requiring supported accommodation, $8 \%$ requiring specialist placement (including forensic), $8 \%$ being discharged to a family members address and $5 \%$ being discharged to temporary accommodation.

Therefore an analysis of variance was conducted to look further into "discharge destination" and post hoc tests showed that there were significant differences in length of stay between the groups being discharged home, to a family address or to temporary accommodation compared to being discharged to inpatient rehabilitation or specialist services, concluding that being discharged to a rehabilitation placement or specialist services (eg secure services) were adding significantly to the length of stay. "Supported accommodation" showed no significant difference between the two groups. 
Discussions concluded that in order to streamline the discharge process and reduce unnecessary delays, intervention would be required as soon as the need for a longer term placement or more intensive support in the community had been identified by the multidisciplinary team.

Following on from this, a discharge coordinator role was established whereby a member of the CRAC team would attend multidisciplinary acute care meetings to discuss care plans and any issues arising that could cause a delay to discharge. In these meetings the team was able to identify whether an individual required a placement earlier and so could intervene more quickly, hopefully resulting in faster discharge from the ward if acute care was no longer required.

The CRAC team also took on the role of gatekeeper for all rehabilitation beds in the local area, meaning that anyone who is identified as requiring longer-term care is assessed and a decision made on whether the team feel that they are suitable for rehabilitation services. Referrals are then made by the team who are able to give detailed accounts of the individuals' needs and circumstances from a rehabilitation perspective.

Through having this specific role, the CRAC team was able to build links with external placement providers. Links to community support networks built through the existing rehabilitation service were also utilised. The team leader also sat on monthly funding panels, liaising closely with commissioners and having a detailed knowledge of the individuals who had been referred.

The overall strategy of having one team to coordinate this process was to streamline care pathways, through more effective communication and having dedicated time to complete paperwork, to reduce any unnecessary delay to length of stay on an acute ward and to facilitate maximum opportunities for people to be placed back into their local community.

\section{Results}

The above data collection continued throughout this process and was analysed using the same methods five months after creating the discharge coordinator and rehabilitation gatekeeper roles. Comparing the data between the two time points shows that during that five months, the time taken for an individual to be discharged to inpatient rehabilitation has reduced by an average of 13.12 days, a specialist placement has reduced by 9.22 days, family address by 2.9 days, and home address by 2.47 . This heavily suggests that the CRAC team have been successful in streamlining care pathways, adding a rehabilitation focus to the acute care pathway for those with complex needs in order to reduce unnecessary delays in hospital.

The CRAC team assess all individuals with a length of stay of over 40 days who do not have a clear discharge plan. The data also showed that overall those who received input (regardless of discharge destination) were discharged on average one day earlier than those who did not require input. This therefore suggests that the team were able to support the multi-disciplinary team to formulate and carry out a discharge plan in the same amount of time as those who already had a clear discharge plan at 40 days, building yet more evidence for the streamlining process taking place.

See supplementary file: ds6164.jpg - "Bar chart showing decrease in length of stay since creating the discharge coordinator and rehabilitation gatekeeper roles"

\section{Lessons and limitations}

The data outlined here looks promising for the CRAC team's role working alongside acute mental health inpatient clinicians, helping to streamline certain processes that are out of the control of the acute care team, for example someone requiring a new placement on discharge. Having one dedicated team to coordinate these processes seems to add value as it improves communication, offers committed time to complete paperwork and may help to reduce lengthy and delayed inpatient stays. However other potential contributing factors cannot be overlooked when discussing explanations for these outcomes.

Before the discharge coordinator role was introduced, the CRAC team were working alongside the acute mental health inpatient clinicians for around a year offering advice and consultation directly on recovery focussed care. In this time the team could have brought more awareness on the time delays of issues such as finding placements, giving the ward staff knowledge on how to address this more quickly and which external providers to approach, meaning work may have already began before the 40 day trigger and the CRAC team becoming involved, helping to further reduce discharge delays.

External factors should also not go unnoticed (for example during the data collection period) it could have been the case that independent placement providers happened to have more empty beds available so there would have been shorter waiting lists; this has not been accounted for in the data. However, it is a time of continuing bed reductions both locally and nationally, as detailed above.

Lastly it should also be noted that this data only concerns those individuals who have had an admission with a length of stay of 40 days or more. The contributing factors for lengthy stays as outlined here have not been compared against those individuals who have required a briefer admission, meaning that the need for streamlining may not just be exclusive to this population. One future area of interest would be to carry out this comparison to understand whether there are wider issues concerning delayed discharge.

\section{Conclusion}

The CRAC team was able to achieve its original aims outlined when first established, to assess and consult on individuals who have had a length of stay on an acute ward for $40+$ days as well as offering intervention and care planning from a rehabilitation perspective; and continues to do so. Much of what the team has added and works 


\section{BMJ Quality Improvement Reports}

towards falls in line with the "good practice toolkit" document discussed earlier, and offers a similar model to the example given from Sheffield Care Trust by creating a discharge coordinator role (Care Services Improvement Partnership: Acute Programme and Change Agent Team, 2007).

Since creating this position, for those who may require a placement and/or a package of care the time taken to be discharged, specifically to a rehabilitation or specialist placement, has reduced. The team continues to grow and provide innovative ideas for new projects, particularly for those individuals with complex needs, supporting them on their road to recovery by focusing on their future goals and aspirations. From being able to produce positive outcomes the team has been showcased as an example of best practice, on both a local and national scale and hopes to continue this way of working into the future.

\section{References}

1. Care Services Improvement Partnership: Acute Programme and Change Agent Team. A Positive Outlook: A Good Practice Toolkit to improve discharge from inpatient mental health care. Wales.nhs.uk. 03 April 2007. Available at: http://www.wales.nhs.uk/sitesplus/documents/829/DoH\%20\%20CSIP\%20Good\%20Practice\%20Toolkit.PDF Accessed on 06 June 2015.

2. Department of Health. No Health Without Mental Health: A Cross-Government Mental Health Outcomes Strategy for People of All Ages. GOV.UK. 02 February 2011. https://www.gov.uk/government/uploads/system/uploads/att achment_data/file/213761/dh_124058.pdf Accessed on 27 November 2014

3. Tyrer P. Has the closure of psychiatric beds gone too far? Yes. BMJ 2011. 343:d7457.

4. McNicoll A. Patients at risk as 'unsafe' mental health services reach crisis point. Community Care. 16 October 2013. http://www.communitycare.co.uk/2013/10/16/patientsat-risk-as-unsafe-mental-health-services-reach-crisispoint-2/ Accessed on 10 October 2014.

5. Care Quality Commission. Better access to safe places in health-based settings for people experiencing a mental health crisis is crucial, urges CQC. Care Quality

Commission. 21 October 2014.

http://www.cqc.org.uk/content/better-access-safe-places-he alth-based-settings-people-experiencing-mental-healthcrisis Accessed on 27 November 2014.

6. Health and Social Care Information Centre. Inpatients formally detained in hospitals under the Mental Health Act 1983 , and patients subject to supervised community treatment. HSCIC. 30 October 2013. http://www.hscic.gov.uk/catalogue/PUB12503/inp-det-m-ha-1983-sup-com-eng-12-13-rep.pdf Accessed 27 November 2014.

7. Keown P, Tacchi MJ, Niemiec S, and Hughes J. Changes to mental healthcare for working age adults: impact of a crisis team and an assertive outreach team. Psychiatric Bulletin 2007. 31:288-92.

\section{Declaration of interests}

Nothing to declare

\section{Acknowledgements}

Clair Jones, Ian Davidson, Linda Shuttleworth

\section{Ethical approval}

This work was approved as a service evaluation (improvement study) through the Trust and was not deemed to require ethics approval as the data used was information previously collected in the course of normal care. 